\title{
A study on the beliefs about the role of physics and physicists amongst introductory students
}

\author{
Miguel Rodriguez ${ }^{1,2}$ and Geoff Potvin ${ }^{1,2}$ \\ ${ }^{1}$ Physics Department, Florida International University \\ ${ }^{2}$ STEM Transformation Institute, Florida International University
}

\begin{abstract}
Modeling instruction in introductory physics has been found to generate positive learning and affective outcomes for students. This study focuses on what students from modeling instruction think about physics, their physics classroom experiences, as well as their perceptions of what it means to be a "physics person", drawing on the lens of identity. We collected data for a four person case study using one-on-one interviews with a purposeful sample of participants nearing the end of their 2 nd semester of physics. The results will motivate a broader study of students' beliefs about the role of physics and physicists and how a modeling instruction experience differentially impacts these beliefs in comparison to a more traditional classroom environment.
\end{abstract}

\section{INTRODUCTION}

Compared to other sciences, physics has seen significantly less growth, in terms of the number of students majoring in physics [1]. The fraction of incoming freshmen who choose to major in physics is less than forty years ago, although the raw number of majors has increased from historic lows in the 1990s [2]. Many ongoing initiatives have attempted to address these issues, without substantial improvement.

Other work has investigated how to improve introductory students' physics understanding, with significant progress in this domain [3,4]. This has led us to more interactive instructional methods; for example, modeling instruction. Modeling instruction in particular has been shown to have attitudinal benefits to the students, increase cognition gains, and improve the performance of unrepresented students [5-7]. However, moving instruction from traditional approaches to more interactive teaching styles has been gradual.

Progress has also been made in understanding students through the lens of physics identity. It has been found that having a strong physics identity is associated with a greater chance of choosing a physics-related career path [8]. The physics identity construct used in this work is built of three related sub-constructs: interest in physics, performance/competence beliefs (beliefs in one's ability to perform and be competent in physics), and recognition beliefs (beliefs that one receives recognition from others as a "physics person"), and has proven to be useful to understanding students' choices and physics persistence [9].
However, one limitation to our understanding in this domain has been assessing what students associate with the role of being a "physics person".

The purpose of the current study was to to probe introductory, modeling instruction-based [3,10] physics students on their beliefs about what being a "physics person" means to them as well as to investigate what their perceptions of physicist and doing physics are. We also want to see how, if at all, their university physics experience relates to those beliefs. The immediate goal is to study how introductory students perceive physics as well as what being a "physics person" entails. The long-term goal of this research is to better understand how students who take modeling physics develop their ideas about physics differently than students who take other physics courses.

\section{METHODOLOGY}

We chose a case study design [11] in order to develop an in-depth view of student perceptions in physics as well as what doing physics and being a physics person means to them. This is the first phase of a planned comparative case study to investigate how student perceptions of physics develop.

In this first phase, four students enrolled in modeling instruction physics courses were selected as cases. We selected these students from the modeling instruction class because they have some experience as to what being a "scientist" is like. We chose a purposeful sample to identify diverse student cases. Specifically, we chose students who were identified as having large gains/losses in physics 
identity during their first semester of physics (by comparison to the mean change amongst students, which was statistically zero). Note that participants had been surveyed twice in the previous semester, pre- and postcourse using an established physics identity survey [9]. The students with physics identity gains are identified here with pseudonyms Jim and Demitri, while the students with physics identity losses are Tia and Nes. Our cases include three men and one woman who were declared as majors of: Biology (Nes), Chemistry (Tia), Mechanical Engineering (Demitri), and Computer Science (Jim). We chose different majors because we were interested in the perspectives of students with varying career intentions and ideas about what being a scientist means. This is also reflective of the typical participants in these courses.

Interviews were conducted near the end of students' $2^{\text {nd }}$ semester of introductory, calculus-based modeling physics course. To conduct the interviews, a semi-structured interview protocol was developed [12]. Interview questions were open ended and in some cases abstract. For example, one of the most relevant questions to this study was "What does it mean to be a physics person?" which often prompted initially vague responses, with subsequent probing helping to elicit further insight. The interview protocol was structured in two phases, the first of which was an accounting of the life history of the participants pertaining to their prior experiences with physics as well as some family and education background. The second phase focused on their perceptions of physics and what being a physics person meant to them. We also asked them about their experiences with university-level physics. We were also interested to find out if they had connected physics concepts to their own lives, or the world around them.

Interviews were played back multiple times and transcribed for subsequent coding. Coding used the package RQDA (R Qualitative Data Analysis) that is part of the R system $[13,14]$. Using RQDA, we developed initial codes using all transcripts and then re-examined the emergent codes into three main themes [15]. Codes were also reviewed between researchers to establish consistency of the coding.

\section{RESULTS}

Two main themes were identified from an analysis of the interviews, which are gathered under the headings: "Physics beliefs and being a physics person" and "Modeling experiences and the relation to perspectives on physics". The first theme, Physics beliefs and being a physics person, encompasses students' likes and dislikes of physics, what it means to do physics, what it means to be a physics person as well as a physicist. The second theme, Modeling experiences and the relation to perspectives on physics, includes past physics background prior to college as well as university-level physics courses. We also mention influences that are a direct result of taking their university physics courses. We report these results because students shape their physics perceptions based on their experience with physics, which mostly come from class. These two themes are built from more specific code categories identified from all the interviews.

A broad result from the transcript analysis is that the students who had physics identity gains, spoke about physics the most and were able to relate it to their careers as well as personal lives more than the two participants who had physics identity losses. Some of the ideas expressed by the participants were directly related to the physics identity sub-constructs (interest, recognition beliefs, and performance/competence beliefs). Next we present a summary of our findings.

\section{A. Physics beliefs and being a physics person}

Results in this theme were primarily derived from responses to the question "What does being a physics person mean to you?" Note that we chose not to use the term physics identity explicitly because it is a theoretical term that has no particular meaning to students, so using the term "physics person" was the closest to what we wanted them to answer [16]. Interest was mentioned by the two student with physics identity gains. Jim said the following:

Being a physics person is someone who is genuinely interested in physics and if someone asks them a question about physics... they can just pull out a piece of paper and be able to do [it].

Here we can also see that he believes that being able to solve physics problems is an essential part of being a physics person. He believes that the ability to solve physics problems is a requirement to be a physics person, which implies a person must perform and be competent about physics. Demitri mentioned interest too, but he believed that seeing connections physics between physics and other things as well as whether a person cares for that knowledge also determines if someone is a physics person.

The students with physics identity losses addressed this issue differently: they framed their answers as being about what it means to be a "physicist" instead. Tia said, "I guess being a physicist is about trying to figure out the unknown."Nes' response aligned with Tia's.

All the students see the role of physicists as researchers who explore the limits of human understanding. Jim said, 
Being a physicist is the career path you take. You are researching new areas of physics that no one else can look into, such as string theory or how does stuff work at the quantum level. Why do things act differently? To me a physicists is like a researcher.

They perceived physics to be a field that is exploring new things and pushing the boundaries of existing human knowledge.

We also asked students about their perceptions of what it means to "do" physics. This is slightly different than the previous question because these students perceive that they had "done physics" (of a kind) due to the way the modeling curriculum is designed. Tia said,

You're trying to break every complex unit to simpler things. Kind of like, when we were talking about the electrons in class. When we were using the tape to determine what would be the electron interactions that were going on, so to help explain it we tried to see the difference between a proton and an electron inside of an atom. Draw a picture or a diagram, something to break it down to get an easy understanding in a greater scale.

This perception resonates for modern physics research; it reflects the problem-solving approach in the modeling instruction curriculum as well.

When we asked participants if they considered themselves to be a physics person, we got expected responses. Jim said "sometimes, depends on the topic" which aligns with his beliefs of what a physics person is, as he feels more comfortable with certain topics. Demitri, having the most physics experience out of all the participants, said he is definitely a physics person. Tia said "hell no" and Nes said no as well.

\section{B. Modeling experience and the relation to perspectives on physics}

The second theme, Modeling experience and the relation to perspective on physics, addresses any responses related to being inside a physics classroom, as reported by participants. Three of the four participants had taken physics prior to the modeling physics courses, so they were asked to compare their experiences. This theme also consists of any influence that studying physics, particularly in modeling instruction, has had on them. They were all overall pleased with the modeling instruction course, though not all felt the same about the subject.

When asked how modeling compares to previous physics courses, Jim said "I can just say it's more conceptual. So that's pretty much the big difference." When talking about her previous physics experiences, Tia said:

In introductory physics, we didn't actually have any hands on experience because all we were given was a book to read out of, which was really boring. One thing is to learn it, but to actually see it, to actually support what you learned actually helps emphasized what you learn, and emphasize those skills.

Demitri said, "In high school we learned through power points." All participants emphasized how prior physics was mostly based on solving physics problems using math, whereas in modeling the concepts were stressed much more heavily and they were given other representations to help them understand how to solve physics problems and build models. This is summed up by one of Jim's statements:

Modeling allows me to see the situation a lot clearer because for example [in] physics 1 you can see stuff, you can look at it happen. But like magnets, I don't see anything, I just see this stuff moving. So these new representations allow me to describe the certain aspects of what's going on.

This answer shows that the representations we use in modeling give students alternative ways of thinking about physics.

When asked about their favorite aspects of modeling, all reported liking the social engagement aspects of the class. Nes expressed,"That's my favorite part, actually being able to connect with everybody else around you."

In modeling, students typically gather together in a circle at the end of an activity to discuss their findings and results using white boards (colloquially called "board meetings"). Three of the four expressed how they really liked the board meetings. Tia said,

I like the board meetings, too, because if there is something I was missing I can find it from other group members and vice versa.

On top of the social aspects of modeling instruction, some liked the fact they were "learning by doing" amongst their group, as Tia described above. Demitri added,

Thats one thing I really like about it, you actually feel like your coming up with it, like you're the one that comes up with it, even though they're already there.

An interesting result is that Demitri identified that his recognition beliefs didn't develop until taking the modeling physics course even though he had two years of high school physics experiences. Through group work and him having a solid background in physics, his peers would come to him for answers, hence increasing his recognition beliefs and physics identity overall 
As far as influences from the class, Tia mentioned how she has used some of the representations they learn from modeling for real world problems. Jim in particular said that he would love to work on a physics engine for a video game, which is something he may not had considered if he didn't feel interested or confident in physics.

\section{DISCUSSION AND CONCLUSIONS}

From the results, two students identified physics interest as a factor to being a physics person, and one of them included performance/competence in his response as well. Although they did not explicitly say that recognition is important to being a physics person, we can see that for Demitri, improved recognition beliefs came from being in the modeling class and being a resource for other students. This provides further motivation for educators to generate more interest for physics in the classroom as well as continue the trend towards research-based instructional methods to improve cognitive gains and student interaction. We also saw that participants believe the role of physicists to be one of a researcher, particularly people that explore the limits of our current knowledge. Lastly, we saw that the participants really connected to the social and hands on approach modeling instruction provides. Demitri was an example of how students are given more opportunities to be recognized by their peers.

This work begins an exploration of students' perceptions of the role held by physicists (and, more broadly, "physics people") and how classroom experiences influence the development of these beliefs. This is the "other side of the coin" for understanding students' physics identities, which

[1] National Center for Education Statistics (2017).

[2] AIP Statistical Research Center (2017).

[3] E. Brewe, Am. J. Phys. 1155 (2008).

[4] R. J. Beichner, J. M. Saul, D. S. Abbott, J. J. Morse, D. Deardorff, R. J. Allain, S. W. Bonham, M. Dancy, and J. Risley, Res. Reform Univ. Phys. 1, 2 (2007).

[5] E. Brewe, L. Kramer, and G. O’Brien, Phys. Rev. Spec. Top. - Phys. Educ. Res. 5, 1 (2009).

[6] J. Jackson, L. Dukerich, and D. Hestenes, Sci. Educ. 17, 10 (2005).

[7] E. Brewe, V. Sawtelle, L. H. Kramer, G. E. O’Brien, I. Rodriguez, and P. Pamelá, Phys. Rev. Spec. Top. Phys. Educ. Res. 6, 1 (2010).

[8] Z. Hazari, G. Sonnert, P. M. Sadler, and M.-C. Shanahan, J. Res. Sci. Teach. 47, 978 (2010).

[9] G. Potvin and Z. Hazari, 2013 PERC Proc. 47, 281 (2013). have been well measured in one sense: having students selfidentify as feeling like "physics people" without necessarily specifying what the role means to the individual.

The limitations of this paper are that the interviews were conducted by the class TA. This may be a limitation

because students may have refrained from making negative comments about the class. However, this is limited because the scope of the interview was broad and not strongly tied to students' performance in physics. Another limitation is that this case study only includes modeling students. The case study design itself means we also cannot make general claims about physics students (or modeling physics students).

Because of the way physics is predominantly taught in many classrooms, students beliefs about physics are limited to certain practices (e.g. often, rote problem-solving) and roles (e.g. solving problems not relevant to every day lives [17]. Modeling instruction provides an alternative set of practices that gives the students a variety of experiences with what being a scientists might be like [3]. We hope to expand this study in the future to include more students from modeling courses as well as other introductory physics courses, to do a comparative case study. We can then assess how physics identity develops in both types learning environments.

\section{ACKNOWLEDGEMENT}

We would like to thank the DBER group in the STEM Transformation Institute at FIU as well as the participants in this study for giving their time and experiences. Without them, this research would not have been possible.

[10] D. Hestenes, Am. J. Phys. 55, 440 (1987).

[11] J. W. Creswell, Qualitative Inquiry \& Research Design: Choosing Among Five Approaches, Third (Thousand Oaks: Sage Publications, 2007).

[12] I. Seidman, Interviewing as Qualitative Research: A Guide for Researchers in Education and the Social Sciences (Teachers College Press, 2006).

[13] R. Huang, (2016).

[14] R Core Team, (2017).

[15] R. E. Boyatzis, Transforming Quantitative Information: Thematic Analysis and Code Development (sage, 1998).

[16] M. Shanahan, M. Nieswandt, E. South, and C. Tg, J. Res. Sci. Teach. 48, 367 (2011).

[17] J. Sweller and Sweller, J., Cogn. Sci. 12, 257 (1988). 